\title{
Study on the geothermal model of Gonghe-Guide region, Qinghai, China
}

\author{
Xueyu Zhao, Zhaofa Zeng, Kun Wang, Nan Huai \\ College of Geoexploration Science and Technology \\ Jilin University \\ Changchun, China
}

\begin{abstract}
The Gonghe basin in north-eastern QinghaiTibetan plateau of China is a potential geothermal field, where there have been some hot springs discovered in recent years. The seismic and magnetotelluric sounding (MT) inversion results in this area show that there are deep crustal fractures which might be channels of the heat production. However, as for the heat source distribution of the region, it is complex. First, it can exclude the granite through the ratio of heat generation testing. Second, the depths of Moho, Curies surface and the epicentral distribution of earthquake show that there is a stable structure in the study area. The substance of this stable structure might come from the southeast part of the Qinghai-Tibetan plateau. Last, the geothermal model is given. It contains the stable structure and the direction of the mantle substance flowing. The model would be helpful for the next geothermal explorations or geophysical work.
\end{abstract}

Keywords-gonghe-guide geothermal field; geothermal resource; geophysics; geothermal model

\section{INTRODUCTION}

The Qinghai-Tibetan plateau is an important geothermal field in China. Gonghe-Guide region is on the northeast part of it, and there are many high temperature springs and geothermal wells discovered in this area (Fig.1). The geothermal prospecting work in Gonghe-Guide basin dated back to the 1990s. To 2013, there were six geothermal wells whose depths were over 1000 meters. And three of them were drilled into the granite layer, the depths of which are 39, 100, 219 meters respectively. The temperature test showed that these granite rocks were hot without any water. Meanwhile there were more than ten shallow geothermal drills whose gradient could be as high as $60-70^{\circ} \mathrm{C} / \mathrm{km}$. In 2014 , the drills at the depth over 3000 meters in Gonghe and those over 2500 meters in Guide were both more than $150^{\circ} \mathrm{C}$. These evidence all show that Gonghe-Guide area is a potential geothermal field ${ }^{[1]}$.

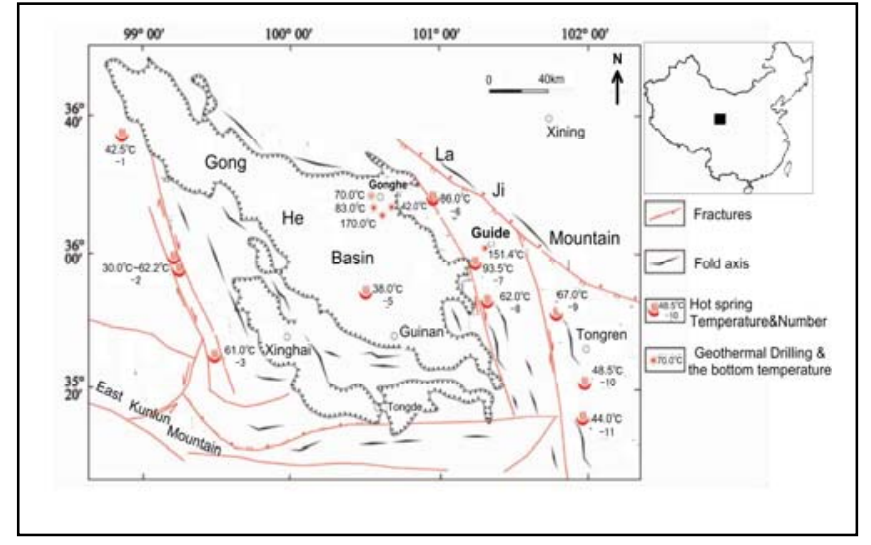

Fig. 1. The locations of drillings and hot springs and the temperature statistic in Gonghe Basin are from ${ }^{[1]}$. The data near the Gonghe City have been updated according to the recent measuring results $\left(70^{\circ} \mathrm{C}, 83^{\circ} \mathrm{C}\right.$, and $\left.170^{\circ} \mathrm{C}\right)$.

\section{THE UNDERGROUND STRUCTURE}

\section{A. Natural Seismic, Earthquake and MT Evidence}

Using receiver function to inverse the seismic velocity structure can show the underground structure. There is a low velocity zone beneath $15-40 \mathrm{~km}$ in the south part of Gonghe Basin, which connects the low velocity zone at the depth of $10 \mathrm{~km}$ and expands to the underground in the middle of Gonghe basin $^{[2-3]}$.

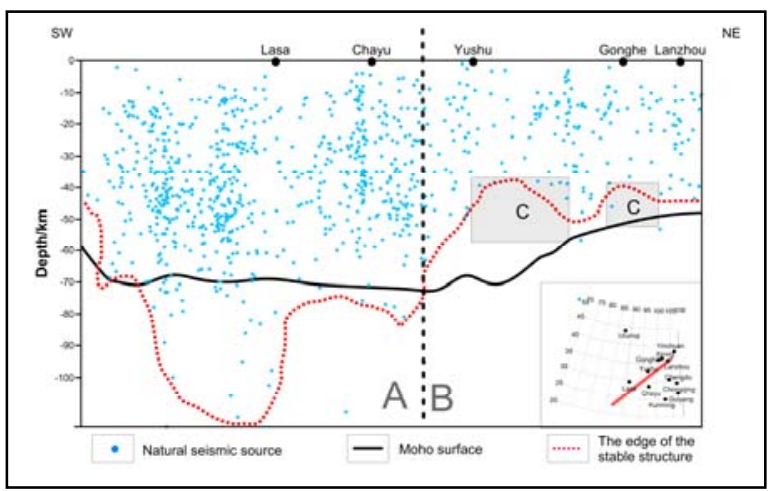

Fig.2 The Epicentral distribution of earthquakes depth are before Oct.2014 from USGS, and Moho depth in the profile from Lasa to Yinchuan is from [4] 
Through MT results, there are the high conductivity layers where the substance might be the combining fused mass and salty fluid. This electrical property contrast in the QinghaiTibetan plateau might be controlled by the collusions between Indian Plate and Eurasian plate. Besides, at the depth of $10 \mathrm{~km}$ of Gonghe Basin, there were low velocity anomalies caused by high temperature in the deep mantle beneath the Eastern Kunlun and the south of Kunlun Mountain to Qingshui River, which showed that the heat resource might have a close connection with the low velocity substance ${ }^{[3]}$.

Besides, it would be helpful to study underground structure if we utilize the epicentral distribution of earthquakes depth. The data is from USGS (U.S. Geological Survey) and we draw it in the profile from Lasa to Lanzhou (Fig 2). It can be obviously seen that the depth varies. It is divided into two areas $\mathbf{A}$ and $\mathbf{B}$. In area $\mathbf{A}$, it is relatively deeper and concentrated, and the deepest one can reach over $100 \mathrm{~km}$. In contrast, it is much shallower and sparser in area B. Especially, in the area $\mathbf{C}$ (belongs to $\mathbf{B}$ ) near Yushu and Gonghe, the depths are almost below 50km. It might be a stable structure (Red dotted line drawn in Fig 2) that makes it less likely for earthquakes to happen in this area.

\section{B. The Moho Depth Change from the Seismic Data}

The Moho surface is an important interface while studying crust and mantle structure.

The Moho surface of the Qinghai-Tibetan plateau could be calculated by using different methods of natural seismic inversion. As for the result, it was deeper in the west and shallower in the east. There is a $40 \mathrm{~km}$ gap between the deepest and shallowest ones. This could show the process of the crust thickness decreasing and increasing caused by the interaction between Indian plate and Eurasian plate, as well as the substance flowing from west to east ${ }^{[4]}$..

\section{The Moho Depth Change from the Gravity Data}

In spite of the seismic way, the Moho depth could be gained from the gravity data as well. Related to a known gravity anomaly, the geometry of a density interface could be computed through some algorithms. The Parker's algorithm is based on the Fourier transform of the gravitational anomaly as a result of the sum of the Fourier transforms of the powers of the surface causing the anomaly. Oldenburg ${ }^{[5]}$ demonstrated that Parker's expression could be rearranged in order to determine the geometry of the density interface from the gravity anomaly. David Go'mez-Ortiz et al. ${ }^{[6]}$ provided the Matlab code.

The gravity data is from the published gravity abnormal map in Qinghai Province, the scale of which is 1:500,000. Compared with the seismic one, it focuses smaller area and the tendency is more obvious. The gravity abnormality ranges from $-530 \mathrm{mGal}$ to $-250 \mathrm{mGal}$, and it is increasing from southwest to northeast. The Gonghe-Guide geothermal field is in the gravity gradient zone.

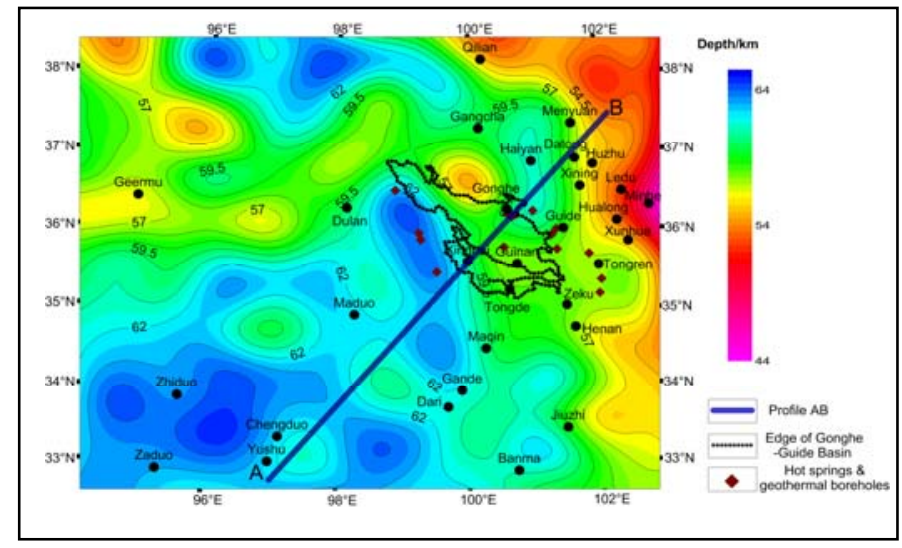

Fig. 3. This moho depth is calculated from the gravity data.

\section{The Curie Interface Depth}

The curie interface is a special isothermal surface where the high temperature can make the substance lose their magnetism. There is a connection between gravity and magnetic anomalies ${ }^{[7]}$. So the method that deals with the gravity data could also be applied to the magnetic data.

The aeromagnetic data is also at the scale of 1:500 000 . And it is higher in the northwest part, while the lower ones that below $-100 \mathrm{nT}$ are disordered.

As for the Curie result, it is relatively low in the study area. In order to make the change of Moho and curies interface obviously, we choose the profile $\mathrm{AB}$ to analyze. This will be discussed later.

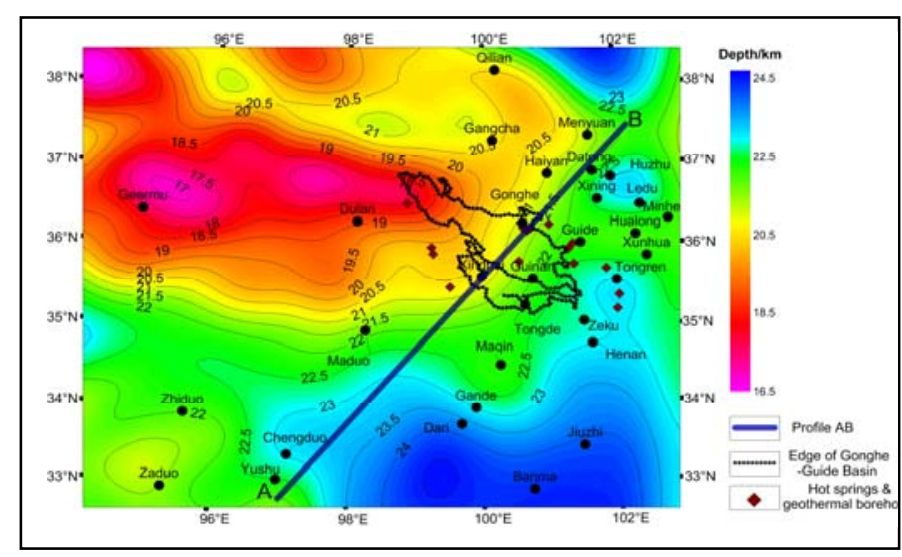

Fig. 4. This Curie interface depth is calculated from the aeromagnetic data..

\section{THE GEOTHERMAL CONDITIONS}

\section{A. The Heat Sources}

The heat source is an important issue to study. There are many kinds, such as the granite which contains radioactive element that can produce much heat. The average ratio of heat generation in the north part of Tibet is $2.6 \pm 1.6 \mu \mathrm{W} / \mathrm{m}^{3}$ and at the medium rate compared to the world average rate. That means, there is no obvious abnormality. Hence, granite could not be the major resource in the study area ${ }^{[8]}$. 
The heat source might originate from the crust and upper mantle. But in terms of the geological condition of Xining Basin which is near our study area, the bedrock depth varied. This might make near surface geothermal display differently and deep heat uniformly distributed.

The terrestrial heat flow from Chadamu to Qilian is 50 to $70 \mathrm{mV} / \mathrm{m}^{2}$, which is at the medium rate. Besides, it is an important condition for magmatic intrusion that the lithosphere is crispy in the area ranging from Chaidamu to Qinghai. There are still tectonic activities such as shear friction caused by continuous extrusion stress field Qaidam fault. The material in the mantle is to Gonghe in horizontal direction in Qinghai-Tibetan plateau ${ }^{[9]}$. And the horizontal pushing force towards north formed by the collision of India plate and Eurasian plate during the Himalayan orogenic period is not completely absorbed by the Qinghai-Tibet Plateau, and the rest of which would be transferred to the Qilian structure belt through the north Kunlun-Qaidam terrain. It makes the temperature of the crust-mantle near Qaidam fault zone increase. In spite of this, in terms of temperature distributions, the zone with thick crust and thin mantle has an abnormal heat crust-mantle structure of "Thick crust and thin mantle", whose formation mechanism is that lithospheric thickens downward as crust thickens, at the same time it destroys the original thermal balance between the boundary of lithosphere and asthenosphere and induces local mantle convection. At last, it leads to the result of melting and thinning of the bottom of lithosphere ${ }^{[10]}$.

\section{B. Fractures in Study Area}

Deep fracture tectonics could control the formation of a heat-conducting channel where the heat flows and diffuses ${ }^{[11]}$. And as major parts of the central orogenic system, Qinling and Kunlun orogens constitute a giant orogenic belt that stretches from east to west in central China. But in Gonghe area they are of discontinuous structure. The size and strength of crustal internal structure, interface undulation and low velocity structure between layers gradually are weaken from south to north along the wide-angle reflection/refraction seismic sounding profile from Luqu to Lanzhou. The contact zone of different structural units of the profile has obvious lateral heterogeneity, where there might exist deep crustal fractures [12].

\section{GEOTHERMAL MODEL}

We get the integrating results in Fig.5. It focuses on smaller area than that in Fig. 2.The overshadow area is our study area. The curve of Curies depth is smooth, and it is relatively shallower in the study area. This can show that the heat from deep does play a role for the geothermal anomaly. The Moho curves fluctuated. Similarly, there is an obvious raise in the area between Tongde and Gonghe. The result of velocity of transverse waves made by ${ }^{[2]}$ also shows that there is a zone protruding according to the line $4.2 \mathrm{~km} / \mathrm{s}$. Meanwhile, the longitudinal wave velocity and MT results show that there is a band abnormality at the depth between 30 and $40 \mathrm{~km}$ beneath Gonghe, where there might be the deep fracture as a passage to transfer the heat. From the discussion of the material flow direction and the geothermal mode, we draw the bold line to show the mantle materials flowing. At the same time Fig 6 shows the abstract geothermal model based on Fig. 5.

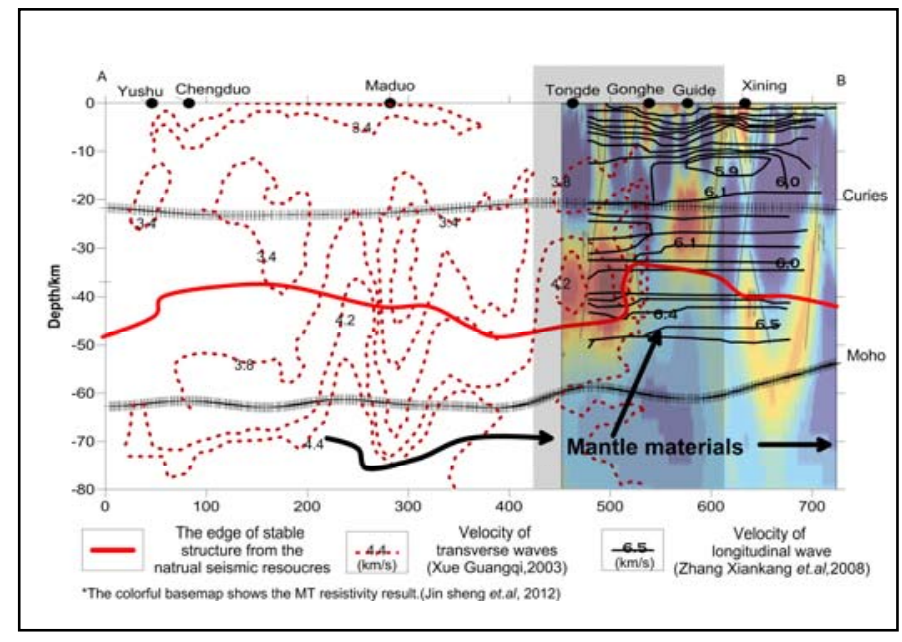

Fig. 5. The profile $\mathrm{AB}$ is marked in figure 3 to 4 . The result of velocity of transverse waves is from ${ }^{[2]}$.

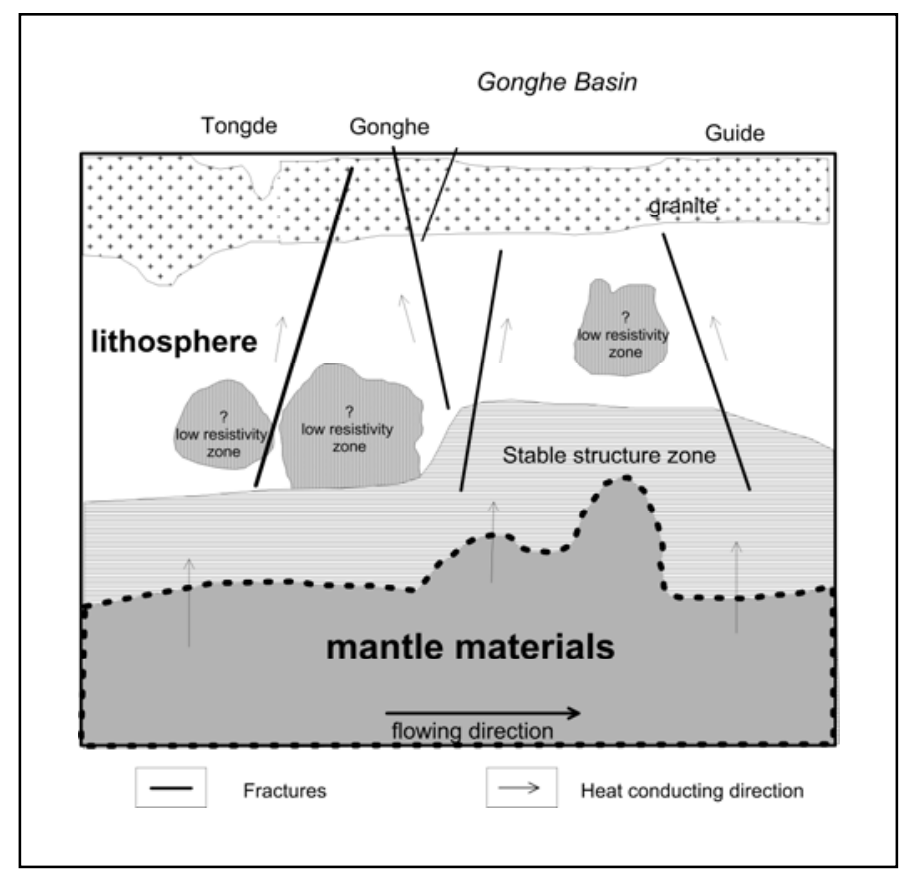

Fig. 6. This abstract geothermal model is based on Fig 5. The upper layer might be the granite. The mantle material and its flowing direction has been shown. However, there are some low resistivity zones which should be discussed more by other geophysical methods.

\section{CONCLUSIONS}

From the geothermal statistics, we know that Gonghe area is a potential geothermal field, and the basis is granite. It is significant to study this area for the future geothermal and hot dry rock study. The geophysical results show that there is a stable structure underground. The geothermal conditions are good in this area. The substance in the mantle from southwest and the melting of the mantle may make contribution to the 
geothermal anomaly, as granite also provides the heat. The fracture might help to transfer the heat from the deep zone. And the geothermal model in this area has been given.

Through this study, we can know that it is a potential area for the future geothermal exploitation, especially for hot dry rock, because its source is from the deep substance. Besides, the way that we study could be helpful for the geothermal prospecting in other areas. For example, Yushu along its neighbor area has the similar geothermal condition, thus it is worth studying in the future.

However, besides the boreholes statistics in the study area, it lacks precise geophysics work, such as smaller scale's MT work and precise seismic work. They are all required in the future geothermal exploitation.

\section{ACKNOWLEDGMENT}

The present study was supported by Doctoral Fund of Ministry of Education of China(20130061110060), the project 863project(2012AA052801)and NSFC grant (41574097).

\section{REFERENCES}

[1] J.Q Xue et al., 2013, Geological-geophysical characteristics of enhanced geothermal system (HDR) in Gonghe-Guide Basin, Geophysical and geochemistry exploration, 37(1) : 35-41.

[2] G.Q Xue, H Qian, M Jiang et al., 2003, Studies on the Velocity Structure of Crust-Upper Mantle beneath Northeast Qinghai-Tibet Plateau, China, ACTA GEOSCIENTIA S INICA, 24(1):19-26
[3] W.D Yan, Y.X Wang, X.Z Gao et al., 2013, Distribution and aggregation mechanism of geothermal energy in Gonghe Basin, Northwest geology, 46(4) : 223-229.

[4] R Gao, X.S Xiong, Q.S Li, Z.W Lu, 2009, The Moho Depth of QinghaiTibet Plateau Revealed by Seismic Detection,Acta Geoscientica Sinica, 30(6):761-773.

[5] D.W. Oldenburg, 1974, The inversion and interpretation of gravity anomalies, Geophysics, 39 (4) (1974):. 526-536

[6] David Go'mez-Ortiz et al. 2005, 3DINVER.M: a MATLAB program to invert the gravity anomaly over a 3D horizontal density interface by Parker-Oldenburg's algorithm, Computers \& Geosciences 31 (2005) 513-520.

[7] Gar land G D.1951 Combined analysis of gravity and magnetic anomalies. Geophysics, 1951, 16(1): 51-62.

[8] X.J Shen, S.Z Yang, J.Y Shen, W.R Zhang, 1989,Experimental Study Of Radiogenic Heat Production Of Granitic Rocks In Tibet, Acta Petrologica Sinica, 4:83-91.

[9] X.X Mo, 2011, Magmatism and Evolution of the Tibetan Plateau, Geological Journal of China Universities, 7(3):.351-367.

[10] S.J Shen, S.Z Yang, J.Y Shen, 1995, Heat Flow Study And Analysis Along the golmud Ejniql geotransect,Chinese J .Geophys. (in Chinese),38 : 86-96.

[11] W.D Shi, S.Q Zhang, N.N Zhou et al. 2006, Distribution characteristics of geothermal water on the northwestern margin of the Xining Basin, Geology in China,33(5) : 1131-1137.

[12] S.X Jia, X.K Zhang, 2008, .Study on the crust phases of deep seismic sounding experiments and fine crust structures in the northeast margin of Tibetan plateau. Chinese J. Geophys.(in Chinese), 51(5):1431-1441. 